\section{Design of liquid- crystalline foods via field theoretic computer simulations}

\author{
Raffaele Mezzenga ${ }^{a, b, *}$, \\ Won Bo Lee ${ }^{c}$ and \\ Glenn H. Fredrickson ${ }^{\mathrm{c}, *}$
}

\author{
${ }^{a}$ Nestlé Research Center, Vers-Chez-Les-Blanc, 1000 \\ Lausanne 26, Switzerland \\ (Tel.: + 4121785 8078; fax: + 4121785 8554; \\ e-mail: raffaele.mezzenga@unifr.ch) \\ ${ }^{b}$ Department of Physics, University of Fribourg, \\ Perolles, Fribourg $\mathrm{CH}-1700$, Switzerland \\ (Tel.: + 4126300 9066; fax: + 4126300 9747; \\ e-mail: raffaele.mezzenga@unifr.ch) \\ ${ }^{c}$ Department of Chemical Engineering, University of \\ California, Santa Barbara, CA 93106, USA \\ (Tel.: + 1805893 8308; fax: + 1805893 8797; \\ e-mail: ghf@mrl.ucsb.edu)
}

Liquid crystalline foods based on self-assembly of lipids and water present rich phase diagrams, in which the hydrophilic and hydrophobic phases are organized in periodic threedimensional nano-structures of different types, depending on temperature and composition. Since these systems constitute a rare example of food at local thermodynamic equilibrium, their structure can be predicted, in theory, based on the minimization of the total free energy. In the present work, we review past and recent attempts to predict the equilibrium structure of these materials, based on parametric, geometrical and physically inspired approaches. We also summarize our own efforts towards a general predictive simulation tool based on self-consistent field theory. For a specific lipid-water system, this approach

* Corresponding authors. allows predicting which liquid crystalline phases are present at equilibrium, but also the relevant topological parameters at a given temperature and composition, such as the radius of water channels or thickness of water lamellae and lipid bi-layers. Furthermore, the addition of guest molecules in either the hydrophobic or hydrophilic phase can also be accounted in the model. This approach should ultimately allow the design of complex liquid crystalline foods based on multiple components and functional molecules.

\section{Introduction}

Liquid crystalline-type foods are nano-structured materials based on the self-assembly of water and short surfactants, typically of lipids family, such as monoglycerides or phospholipids (Krog, 1990). They can be found, for example at the water-oil interface on salad dressing and other types of emulsions. When regarded as a material itself, these systems are of interest for a number of applications in food science and technology. When re-dispersed in water in the form of colloidal systems with an internal structure, they can be used as efficient carriers of hydrophobic compounds, such as aroma and flavors, and within the limits dictated by osmotic gradients, can also be loaded by hydrophilic compounds such as peptides or saccharides (de Campo et al., 2004; Lindstrom, Ljusberg-Wahren, Larsson, \& Borgstrom, 1981; Mariani, Rustichelli, Saturni, \& Cordone, 1999; Mezzenga et al., 2005; Saturni, Rustichelli, Di Gregorio, Cordone, \& Mariani, 2001). When used in their bulk form, they constitute efficient building blocks for more complex functional foods, or can be employed as 'nanoreactors' to run and control food-specific chemical reactions within confined geometries (Vauthey et al., 2000). Selfassembled lipids and water have very rich phase diagrams, where exact boundaries depends on the type of lipid used, but which in general include isotropic fluid $\left(\mathrm{L}_{\mathrm{II}}\right)$, lamellar phases with amorphous $\left(\mathrm{L}_{\alpha}\right)$ or crystalline lipid domains $\left(\mathrm{L}_{\mathrm{C}}\right)$, inverted columnar hexagonal cylinders $\left(\mathrm{H}_{\mathrm{II}}\right)$, as well as bicontinuous double gyroid (Ia3d), double diamond $(\mathrm{Pn} 3 \mathrm{~m})$, and primitive $(\mathrm{Im} 3 \mathrm{~m})$ cubic phases (de Campo et al., 2004; Mezzenga et al., 2005; Qiu \& Caffrey, 2000). Experimental techniques such as small angle $\mathrm{X}$-ray scattering (SAXS) or cryo-TEM have allowed extensive and detailed characterization of the topology and structure of the most relevant liquid crystalline phases encountered 

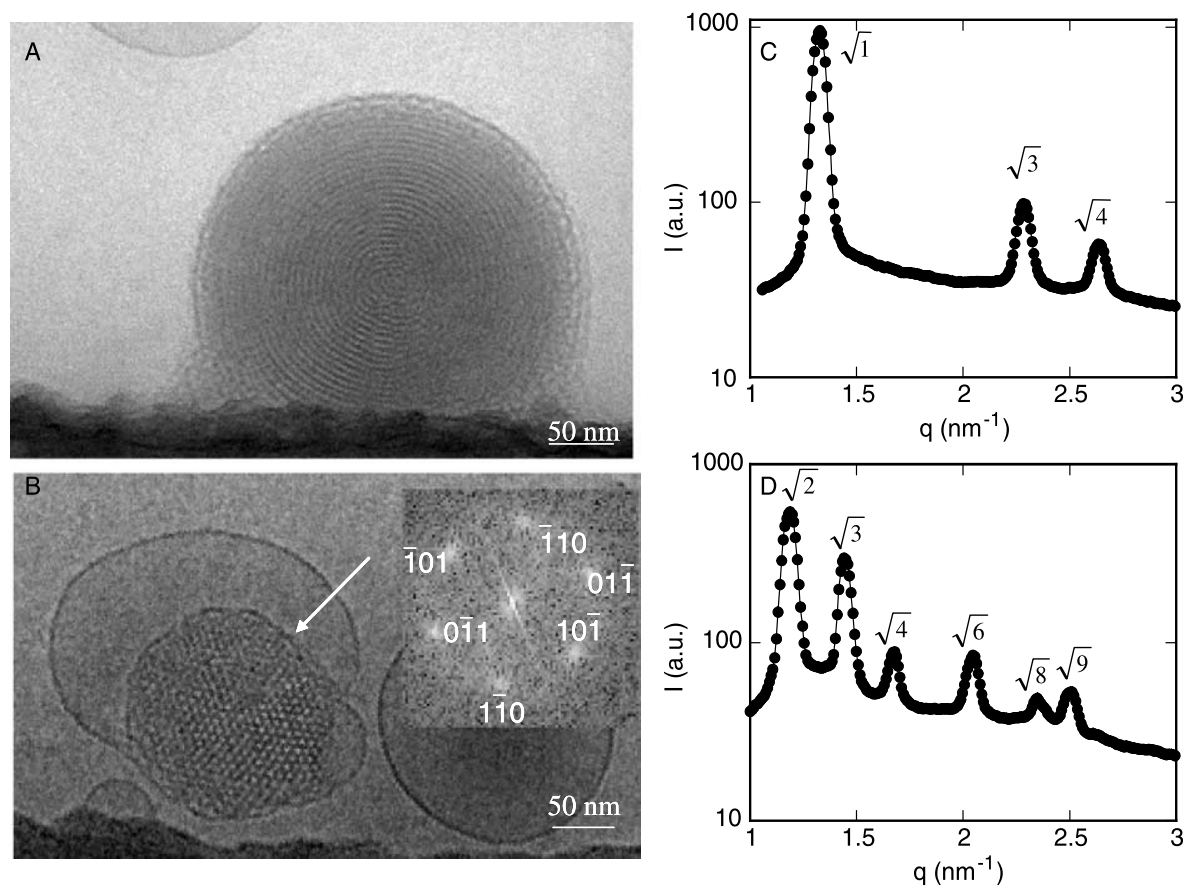

Fig. 1. (a) Examples liquid crystalline phases based on self-assembled monoglycerides and water. Cryo-TEM images of (a) $H_{\|}$and (b) Pn3m liquid crystalline phases re-dispersed in water (from de Campo et al., 2004; Yaghmur et al., 2005). Small angle X-ray scattering spectra of homologue bulk liquid crystalline structures: (c) bulk $\mathrm{H}_{\|}$and (d) bulk Pn3m phases (from Mezzenga et al., 2005).

in foods. Fig. 1 compares the cryo-TEM images of $\mathrm{H}_{\mathrm{II}}$ and Pn3m liquid crystalline foods re-dispersed in water, with the SAXS data obtained in the homologue bulk liquid crystalline phases (de Campo et al., 2004; Mezzenga et al., 2005; Yaghmur et al., 2005).

As far as physical properties, rheology has been the most extensively used technique to characterize liquid crystalline foods. Depending on the specific mesophases investigated, very different rheological properties can be found for the same mixture of lipid and water. Thus for example, in the same lipid-water formulation, purely plastic materials (lamellar), viscoelastic fluids (hexagonal) or strong rigid structures (cubic), with storage moduli in the range $10-10^{6} \mathrm{~Pa}$ (Mezzenga et al., 2005; Pitzalis et al., 2000) can be found, and the final properties of the food based on that specific recipe will be greatly affected by the equilibrium mesophase structure. The rigidity of cubic phases $\left(\sim 10^{6} \mathrm{~Pa}\right)$, as an example, is remarkably high when bearing in mind the 'soft' nature of the components. Although the high storage modulus of cubic phases is mostly to be attributed to the interfacial tension of the interpenetrated lipid-water interfaces, the exact physical mechanisms responsible for the complex viscoelastic behavior of cubic phases are still debated (Dhinojwala \& Granick, 1997; Granick, 1991; Hu \& Granick, 1992; Jones \& McLeish, 1995, 1999; Mezzenga et al., 2005; RodriguezAbreu, Garcia-Roman, \& Kunieda, 2004). Nevertheless, understanding and tuning the conditions such that a food is mostly composed of bicontinuous cubic phases is equivalent to tuning its respective rheological properties. Finally, we observe that when a specific liquid crystalline food is loaded with an additional component in either the hydrophilic or hydrophobic phase, as it is the case for complex functional foods, order to order transitions from one liquid crystalline phase to another are routinely observed (Mezzenga et al., 2005). Thus, in analogy with other areas of soft condensed matter, the study of liquid crystalline foods would greatly benefit from the existence of a predictive tool, allowing the determination of the most stable phase under controlled conditions of temperature, $\mathrm{pH}$ and composition. Such a tool would then permit the design not only of specific liquid crystalline phases, but also the physical properties of foods.

\section{Predicting phase diagrams: background}

The thermodynamics ruling the self-assembly and structures of liquid crystalline foods has been investigated for more than 30 years, but it remains widely debated. Although experimental techniques suitable for the investigation of these systems are well established and have provided detailed information on the structures of the most commonly observed phases (Monduzzi, Ljusberg-Wahren, \& Larsson, 2000; Qiu \& Caffrey, 2000; Thies et al., 2002), a general theoretical tool allowing quantitative prediction and interpretation of both the thermotropic and lyotropic behavior of these materials is still to be developed. The general physical mechanisms responsible for self-assembly are well understood, but the complexity of the physics hinder quantitative analysis. Despite their low molecular weight, lipids and water self-assemble into ordered structures owing to the very large enthalpy of mixing 
favoring the formation of two domains composed of (i) a mixture of water and the hydrophilic lipid heads and (ii) the hydrocarbon based lipid tails. By measuring the activity of alkanes partitioning in water at room temperature, and using Flory-Huggins formalism, this enthalpy can be estimated to be of the order of $\chi \approx 3$ at room temperature (Mezzenga, Schurtenberger, Burbidge, \& Michel, 2005). Considering that for macromolecular amphililes (block copolymers) the order-disorder transition occurs when $\chi \times$ $N \approx 10.5$ (where $N$ is the number of segments of the polymer backbone) (Hamley, 1998), one can very well appreciate that in the case of lipids, the threshold in molecular weight of the surfactants for self-assembly is $N \approx 3-4$ segments, which is equivalent to surfactants of the order of 12-16 carbon atoms. Here, one must recognize that for hydrocarbon chains, the number of carbon atoms is approximately 4 times the number of statistical segments $N$ (Cochran \& Bates, 2002). Although quite rudimentary, this argument illustrates well why in foods, where typical lipid-based surfactants have more than 12 carbons, self-assembly is regularly observed. Despite this simplistic vision, theoretical approaches aimed at the prediction of phase diagrams in lipid-water systems, have been very unsuccessful compared to other close fields of soft condensed matter, such as for example the self-assembly of block copolymers.

A simple approach still commonly used for a first rough classification of the various structures of liquid crystalline foods is based on the concept of critical packing parameter (CPP) (Israelachvili, 1991). This is expressed geometrically as the ratio between the volume of the hydrophobic lipid tail, $v$, and the product of the cross-sectional lipid head area, $A$, and the lipid chain length, $l$. Thus, different liquid crystalline phases may be predicted based on the curvature of the water-lipid interface. For example $\mathrm{L}_{\alpha}$, cubic phases, and $\mathrm{H}_{\mathrm{II}}$, are expected when the CPP has a value of $v /(A l) \approx 1$; $v /(A l) \geqq 1$ and $v /(A l)>1$, respectively (Israelachvili, 1991; Jonsson, Lindman, Holmberg, \& Kronberg, 2001). The CPP has shown some success in explaining qualitative features and tendencies of thermotropic liquid crystalline foods. For instance, by raising temperature, the number of water molecules hydrating the polar heads of the lipids decreases, due to partial breaking of hydrogen bonds. As a result $\mathrm{A}$ decreases and the CPP increases, inducing a cubic to $\mathrm{H}_{\mathrm{II}}$ transition, in agreement with experimental observations (Qiu \& Caffrey, 2000). Analogous arguments can be used to explain the $\mathrm{L}_{\alpha}$ to cubic transition. Nonetheless, the approach based on CPP remains generally qualitative; it can neither fully describe liquid crystal phase diagrams nor provide detailed insight into the topology of complex structures.

Advances in the description of topology of bicontinuous cubic phases have been made by applying triply periodic minimal surface concepts (Scriven, 1976). Ia3d, Pn3m and $\operatorname{Im} 3 \mathrm{~m}$ cubic phases can then be described as threedimensional, periodic lipid bilayers with zero mean curvature at each point of the water-lipid interface. This is consistent with the physical requirement of minimizing the interfacial energy at fixed volume fraction, although the frustration of packing lipid chains is not fully accounted for in the minimal surface representation (Schwarz \& Gompper, 2001). The approach allows for the determination of relevant topological parameters in cubic phases, such as the radius of water channels and the cross-sectional thickness of the lipid bilayer. The theory's major drawback relies on its parametric rather than molecular character, which is neither suitable to microscopic interpretation of self-assembly mechanisms nor can be used to describe phase diagrams of charged/neutral lipid-water systems.

A general physically inspired approach to quantitatively describing the self-assembly of lipids and water has been recently proposed by Li and Schick (2001) and Muller and Schick (1998). As a direct analogy from the polymer physics world, where similar models have been successfully applied to a number of different systems, the model is based on a meanfield approach, in which each lipid chain experiences a mean field generated by all other surrounding chains. Despite the great flexibility of this powerful method, in the case of lipidwater mixtures, the quantitative agreement remains very limited. In the approach proposed by Schick and co-workers, lipid tails, are modeled with the rotational isomeric state (RIS) model (Flory, 1956), which can describe short lipid tails with unsaturated bonds. Therefore, the main reason for the limited quantitative agreement may be an inadequate treatment of the hydrogen bond effect. In amphiphilic lipids, the polar head/ lipid tail enthalpy of mixing increases with temperature, owing to the breaking of hydrogen bonds. This trend differs strongly from the majority of synthetic polymer-solvent pairs, and is responsible for the existence of a lower critical solubility temperature in water-soluble polymers (Bekiranov, Bruinsma, \& Pincus, 1997; Matsyama \& Tanaka, 1990). Additional oversimplifications in the model arise from the neglect of the associating character and the 'hydrophobic effect' connected to the aqueous solvent. In the following sections, we describe our initial attempts towards correcting these deficiencies and implementing a predictive tool, based on self-consistent field theory, for describing the physics of self-assembly in lipidwater systems.

\section{Field theoretic computer simulations}

Coarse grained field theoretic models solved within the mean-field approximation, often referred to as selfconsistent field theory (SCFT) (De Gennes, 1969; Edwards, 1965), have been successfully applied to various polymer systems such as melts, blends, and solutions (Fredrickson, in press; Fredrickson, Ganesan, \& Frolet, 2002; Matsen, 1995a,b; Matsen \& Bates, 1996; Matsen \& Schick, 1994). A particular highlight is the mean-field phase diagram for diblock copolymer melts produced by Matsen (1995) and Matsen and Schick (1994), which represents one of the great successes of SCFT. The theoretical predictions are remarkably similar to the experimental phase diagram (Khandpur et al., 1995), both qualitatively and quantitatively. This shows that SCFT is a solid framework for 
describing the equilibrium self-assembly of complex fluids that possess structured lyotropic and thermotropic phases.

A mesoscopic field-based polymer model is often derived from a particle-based model, which uses positions and momenta of particles as the degree of freedom while a fieldbased model utilizes continuous fields. Once a particle-based model is made, there are formally exact methods for transforming it into a field-based model and by this particleto-field transformation, a many interacting chain problem is decoupled into several single chain problems in the presence of the external fields. The result of the transformation is functional integrals with regard to several fields. Since these functional integrals for any non-trivial model do not have an analytic solution in closed form, numerical methods have to be used (Fredrickson, in press; Fredrickson et al., 2002).

For the purpose of illustration, we introduce the SCFT formalism in the context of a blend of $\mathrm{AB}$ diblock copolymer and A homopolymer using the continuous Gaussian chain model. This system has some common features with lipidwater mixtures since a lipid has the same structure and role as an $\mathrm{AB}$ diblock copolymer, notwithstanding the shorter chain length, presence of unsaturated bonds, and rigid head group. Similarly, A homopolymer can be viewed as a 'solvent' for the A blocks of the copolymer, analogous to the role that water plays in lipid-water systems. A summary of the SCFT equations for this system is displayed in Box 1.

\section{Box 1. SCFT of an incompressible AB diblock copolymer and A homopolymer blend}

We adopt a mesoscopic 'particle' model in which a harmonic stretching energy is used to describe bonded interactions of segments and non-bonded interactions, analogous to those in the Flory-Huggins lattice theory, are described by a $\chi$ parameter. By means of a HubbardStratonovich transformation, this particle-based model is transformed into a field theory. The mean field approximation (or SCFT) is based on the assumption that one specific field configuration, the so-called 'saddle point', dominates the partition function. This approximation implies the following SCFT equations (Fredrickson, in press; Fredrickson et al., 2002; Matsen, 1995a,b):

$H_{\mathrm{G}}=\frac{1}{V} \int \mathrm{d} \underline{r}\left\{\chi_{\mathrm{AB}} N \phi_{\mathrm{A}} \phi_{\mathrm{B}}-\left(W_{\mathrm{A}} \phi_{\mathrm{A}}+W_{\mathrm{B}} \phi_{\mathrm{B}}\right)\right\}-Q_{\mathrm{C}}\left[W_{\mathrm{A}}, W_{\mathrm{B}}\right]-z_{\mathrm{h}} Q_{\mathrm{h}}\left[W_{\mathrm{A}}\right]$

$\phi_{\mathrm{A}}(\vec{r})=\frac{\delta Q_{\mathrm{C}}\left[W_{\mathrm{A}}, W_{\mathrm{B}}\right]}{\delta W_{\mathrm{A}}(\vec{r})}+z_{\mathrm{h}} \frac{\delta Q_{\mathrm{h}}\left[W_{\mathrm{A}}\right]}{\delta W_{\mathrm{A}}(\vec{r})}=\int_{0}^{f} \mathrm{~d} s q_{\mathrm{c}}(\vec{r}, 1-s) q_{\mathrm{c}}(\vec{r}, s)+z_{\mathrm{h}} \int_{0}^{\alpha} \mathrm{d} s q_{\mathrm{h}}(\vec{r}, \alpha-s) q_{\mathrm{h}}(\vec{r}, s)$

$\phi_{\mathrm{B}}(\vec{r})=\frac{\delta Q_{\mathrm{c}}\left[W_{\mathrm{A}}, W_{\mathrm{B}}\right]}{\delta W_{\mathrm{B}}(\vec{r})}=\int_{f}^{1} \mathrm{~d} s q_{\mathrm{c}}(\vec{r}, 1-s) q_{\mathrm{c}}(\vec{r}, s)$

$W_{\mathrm{A}}(\vec{r})=\chi_{\mathrm{AB}} N \phi_{\mathrm{B}}(\vec{r})+P(\vec{r})$

$W_{\mathrm{B}}(\vec{r})=\chi_{\mathrm{AB}} N \phi_{\mathrm{A}}(\vec{r})+P(\vec{r})$

$\phi_{\mathrm{A}}(\vec{r})+\phi_{\mathrm{B}}(\vec{r})-1=0$

where,

$Q_{\mathrm{c}}=V^{-1} \int \mathrm{d} \vec{r} q_{\mathrm{c}}(\vec{r}, 1)$

$Q_{\mathrm{h}}=V^{-1} \int \mathrm{d} \vec{r} q_{\mathrm{h}}(\vec{r}, \alpha)$

$\frac{\partial}{\partial s} q_{\mathrm{c}}(\vec{r}, s)=\left\{\begin{array}{ll}\frac{N b^{2}}{6} \nabla^{2} q_{\mathrm{c}}(\vec{r}, s)-W_{\mathrm{A}}(\vec{r}) q_{\mathrm{c}}(\vec{r}, s), & s<f \\ \frac{N b^{2}}{6} \nabla^{2} q_{\mathrm{c}}(\vec{r}, s)-W_{\mathrm{B}}(\vec{r}) q_{\mathrm{c}}(\vec{r}, s), & f<s\end{array} \quad\right.$ with $\quad q_{\mathrm{c}}(\vec{r}, 0)=1$

$\frac{\partial}{\partial s} q_{\mathrm{h}}(\vec{r}, s)=\frac{N b^{2}}{6} \nabla^{2} q_{\mathrm{h}}(\vec{r}, s)-W_{\mathrm{A}}(\vec{r}) q_{\mathrm{h}}(\vec{r}, s) \quad s<\alpha \quad$ with $\quad q_{\mathrm{h}}(\vec{r}, 0)=1$

$H_{\mathrm{G}}$ is a dimensionless grand canonical potential (Matsen, 1995). $\phi_{\mathrm{A}}(\vec{r})$ and $\phi_{\mathrm{B}}(\vec{r})$ are volume fraction fields of $\mathrm{A}$ and $\mathrm{B}$ segments. $W_{\mathrm{A}}(\vec{r})$ and $W_{\mathrm{B}}(\vec{r})$ are chemical potential fields for $\mathrm{A}$ and $\mathrm{B}$ segments. $z_{\mathrm{h}}$ is the activity of $\mathrm{A}$ homopolymer. $Q_{\mathrm{C}}\left[W_{\mathrm{A}}, W_{\mathrm{B}}\right]$ and $Q_{\mathrm{h}}\left[W_{\mathrm{A}}\right]$ are singlechain partition functions of $\mathrm{AB}$ diblock copolymer and $\mathrm{A}$ homopolymer, respectively, interacting with the chemical potential fields $W_{\mathrm{A}}$ and $W_{\mathrm{B}} . f$ is the volume fraction of the A block on each copolymer and $N$ is the polymerization index of each $\mathrm{AB}$ diblock copolymer. $\alpha$ is the ratio of the polymerization index of an A homopolymer to that of an $\mathrm{AB}$ diblock copolymer. The 'chain propagators' $q_{\mathrm{c}}(\vec{r}, s)$ and $q_{\mathrm{h}}(\vec{r}, s)$, are related to the probability density that a chain of a contour length $s$ has its end at position $\vec{r}$ (Fredrickson, in press; Sides \& Fredrickson, 2003). $q_{\mathrm{c}}(\vec{r}, s)$ and $q_{\mathrm{h}}(\vec{r}, s)$ satisfy the diffusion equations, Eqs. (9) and (10) (De Gennes, 1969; Edwards, 1965; Freed, 1972). 
In order to solve these mean field equations, Eqs. (2)-(6) in Box 1, various numerical methods can be applied (Fredrickson, in press; Matsen, 1995b; Matsen \& Schick, 1994; Sides \& Fredrickson, 2003). Once the modified diffusion equations, Eqs. (9) and (10), are solved, Eqs. (2) and (3) are evaluated. In order to satisfy the remaining equations, Eqs. (4)-(6), $W_{\mathrm{A}}(\vec{r}), W_{\mathrm{B}}(\vec{r})$, and $P(\vec{r})$ are adjusted by means of various numerical convergence schemes (Ceniceros \& Fredrickson, 2004; Sides \& Fredrickson, 2003).

The simulation procedures summarized above are based on the assumption that a simulation box is fixed to a particular shape and volume. However, a fixed shape box produces a residual stress and this stress causes various defects, which should be avoided in order to attain true equilibrium structures. Therefore, another method is required for obtaining stress-free structures, which is crucial for the accurate determination of phase boundaries. Recently, Barrat, Fredrickson, and Sides (2005) reported a variable cell shape method for field theoretic simulations in order to achieve stress-free conditions using a $n \tau T$ ensemble and a parallelepiped cell shape. $\underline{\tau}$ is a thermodynamic tension tensor related to an external $\overline{=}$ stress. Since the system is incompressible, this ensemble maintains a constant total cell volume. Alternatively, we can adjust the total volume, while maintaining a constant segment density, in a separate procedure, where the unit cell size and the unit cell shape are adjusted to minimize an intensive energy density (Fredrickson, in press). The latter is more useful for the unit cell calculations that are described here. Following Fredrickson (in press) and Fredrickson, Barrat et al. (2005), a cell shape tensor $h$, constructed from the three vectors that define the edges $\overline{\bar{o}}$ the parallelepiped simulation cell, is relaxed by means of the following fictitious dynamics scheme

$\frac{\mathrm{d}}{\mathrm{d} t} \underline{=}=-\lambda_{\mathrm{h}} \beta \underline{\underline{h}}\left(\underline{\underline{\underline{\Sigma}}} \mathrm{c}\left[W_{\mathrm{A}}, W_{\mathrm{B}}, \underline{\underline{g}}\right]+\underline{\underline{\underline{ }}}_{\mathrm{h}}\left[W_{\mathrm{A}}, \underline{\underline{g}}\right]\right)$

where $\underline{g}$ is a metric tensor defined as $\underline{g} \equiv h^{\mathrm{T}} h$ and $\lambda_{\mathrm{h}}$ is a relaxation parameter. $\underline{\underline{\Sigma}}_{\mathrm{c}}$ and $\underline{\underline{\Sigma}}_{\mathrm{h}}$ represent internal stresses produced by $\mathrm{AB}$ diblock copolymers and A homopolymers, respectively. These internal stresses vanish at equilibrium when stress-free structures are obtained. The tensors can be expressed in terms of the $q_{\mathrm{c}}$ and $q_{\mathrm{h}}$ propagators in the grand canonical ensemble by invoking a factorization of the single chain path integrals (Barrat et al., 2005; Doi \& Edwards, 1988):

$$
\begin{aligned}
& \underline{\underline{\Sigma}}_{\mathrm{c}}\left[W_{\mathrm{A}}, W_{\mathrm{B}}, \underline{\underline{g}}\right]=\frac{2 R_{\mathrm{c}, \mathrm{go}}^{2}}{\beta} \int \mathrm{d} \vec{X} \int_{0}^{1} \mathrm{~d} s q_{\mathrm{c}}(\vec{X}, s) \underline{\underline{g}}^{-1} \nabla_{\vec{V}} \nabla_{\vec{V}} \underline{\underline{g}}^{-1} \\
& \times q_{\mathrm{c}}(\vec{X}, 1-s) \\
& \underline{\underline{\Sigma}}_{\mathrm{h}}\left[W_{\mathrm{A}}, \underline{\underline{g}}\right]=\frac{2 z_{\mathrm{h}} R_{\mathrm{c}, \mathrm{go}}^{2}}{\beta} \int \mathrm{d} \vec{X} \int_{0}^{\alpha} \mathrm{d} s q_{\mathrm{h}}(\vec{X}, s) \underline{\underline{g}}^{-1} \nabla_{\vec{V}} \nabla_{\vec{V}} \underline{\underline{g}}^{-1} \\
& \times q_{\mathrm{h}}(\vec{X}, \alpha-s)
\end{aligned}
$$

In these expressions, $\vec{X}$ is a cell-scaled position vector whose components lie in $[0,1]$.

By applying these schemes and deterministic initial conditions that 'seed' the $W$ fields to produce a unit cell of a desired mesophase, stress-free structures are obtained including the various bicontinuous cubic phases. Phase boundaries are calculated by comparing the grand canonical potentials between phases at equal values of the homopolymer activity $z_{\mathrm{h}}$. Fig. 2 shows examples of equilibrated stress-free phases for the model AB diblock copolymer-A homopolymer blend described above.

These numerical results show the ability of the SCFT framework to capture the complexity of three-dimensional self-assembly in a block copolymer-homopolymer alloy that is conceptually similar to food grade lipid-water mixtures. The technical challenge of implementing SCFT for the latter types of systems does not lie in either the theoretical framework or the numerical methods, but rather in accurate parameterization of the more complicated interactions and short-scale molecular details that are present in lipid-water mixtures. Specific complicating factors in these systems are the presence of hydrogen bonding among hydrophilic heads and water molecules, unsaturated bonds in the hydrocarbon tails, and short tail length. Muller and Schick (1998) studied the equilibrium self-assembly of a simple model of glycerolmonoolein in water using SCFT techniques. The rotational isomeric state (RIS) model for representing a tail structure was applied and the head of glycerolmonoolein was treated as a rigid rod. To obtain the single lipid chain partition function at fixed field, analogous to the functions $Q_{\mathrm{c}}$ and $Q_{\mathrm{h}}$ above, Monte Carlo simulations were applied. This stochastic method is computationally very expensive (in comparison with the deterministic method of computing partition functions described above), which made determining phase boundaries difficult. Although the RIS model of Muller and Schick described the lipid tail conformations quite realistically, including the presence of unsaturated bonds, their results found limited agreement with the experimental phase diagram (Muller \& Schick, 1998; Qiu \& Caffrey, 2000). It seems likely that the neglect of specific interactions in the model related to head group hydration and hydrogen bonding is responsible for this discrepancy. Nevertheless, the Muller-Schick work is an impressive first step towards extending SCFT methods to food grade systems.

Since hydrogen bonding cannot be described by a simple 'contact' interaction potential, such as embodied in FloryHuggins treatments of polymer solutions and alloys, a more sophisticated treatment of the head group-water interactions is clearly required. One promising approach is the 'm clusters' model of Matsyama and Tanaka (1990) for explaining lower critical solution temperature in polymer solutions and the existence of closed loop phase diagrams in water-soluble polymers such as polyethylene oxide. This model accounts for two important features of hydrogen 

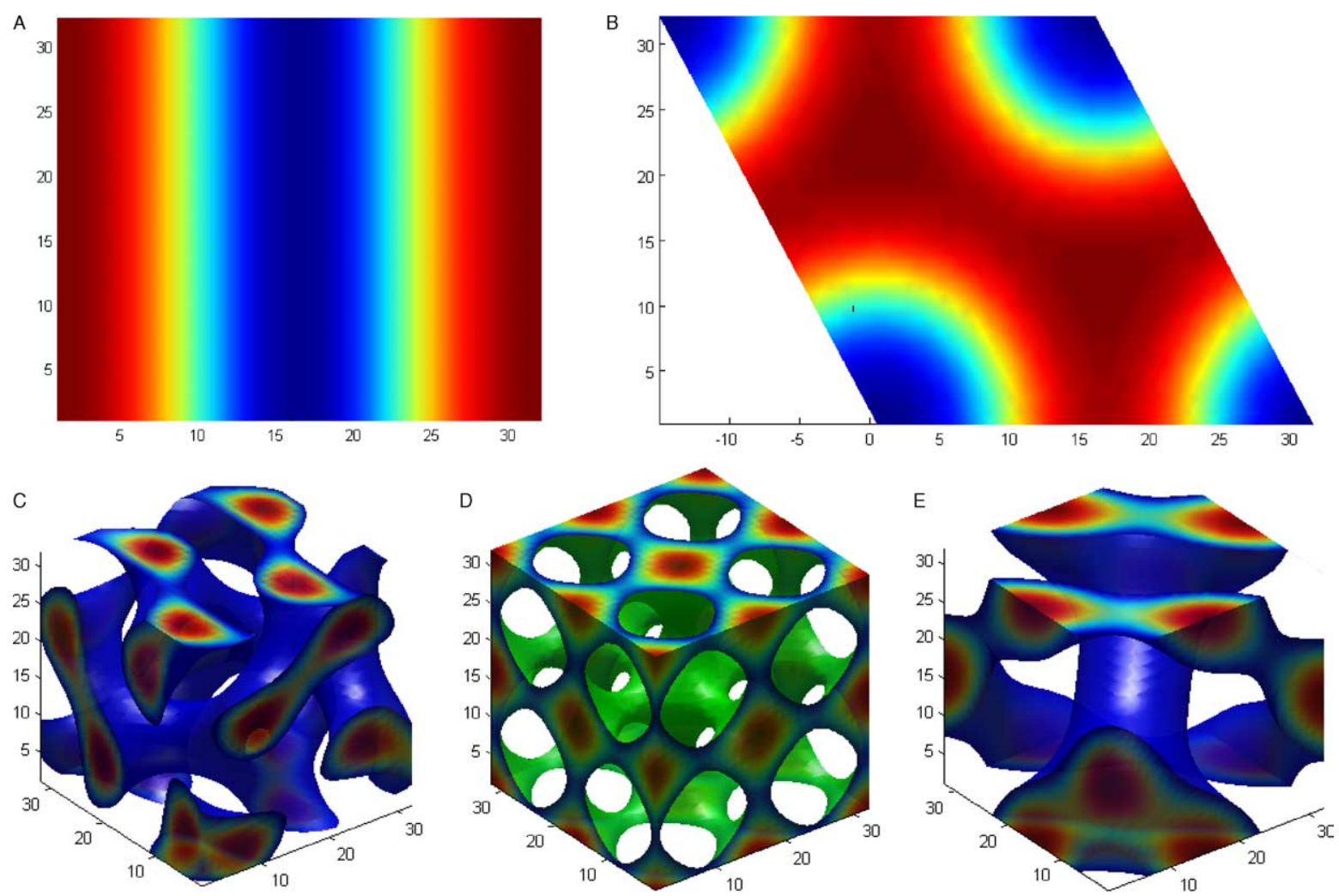

Fig. 2. Lamellar, hexagonal and various bicontinuous cubic phases from SCFT simulations with $\chi \times N=11.0, \alpha=0.67$ and $B_{A}=B_{B}$. Calculations were performed pseudo-spectrally using 32 (Mezzenga et al., 2005) plane waves (basis functions) for lamella and hexagonal phases and 32 (de Campo et al., 2004) plane waves for the bicontinuous cubic phases. (a) Lamellar phase with $f=0.5, z_{\mathrm{h}}=0.15$ and cell side length $L=3.83 R_{\mathrm{c}, \mathrm{go}}$; (b) Hexagonal phase with $f=0.5, z_{\mathrm{h}}=0.17$ and $L=4.83 R_{\mathrm{c}, \mathrm{go} ;}$ (c) Double gyroid cubic phase (la $\overline{3} \mathrm{~d}$ ) with $f=0.5, z_{\mathrm{h}}=0.15$ and $L=9.83 R_{\mathrm{c}, \mathrm{go}}$; (d) Primitive cubic phase $(\operatorname{Im} \overline{3} \mathrm{~m})$ with $f=0.585, z_{\mathrm{h}}=0.11$ and $L=6.83 R_{\mathrm{c}, \mathrm{go}} ;(\mathrm{e})$ Double diamond cubic phase $(\operatorname{Pn} \overline{\mathrm{m}})$ with $f=0.5, z_{\mathrm{h}}=0.15$ and $L=6.05 R_{\mathrm{c}, \mathrm{go}}$.

bonding - the specific on-off nature of the bonds and the fact that available bonding sites can saturate.

Another important development that will be required for the extension of SCFT methods to food grade polymers involves the lipid tail model. While the RIS model is attractive for describing such systems, in part because it allows for the controlled incorporation of unsaturated bonds, stochastic sampling of the RIS single-chain partition function is prohibitively expensive. We believe that deterministic 'transfer matrix' methods (analogous to discrete time approximations for time-stepping the diffusion equations (9) and (10) for the continuous Gaussian chain model) can be developed for the RIS model, as well as for other models that may be useful in describing short, stiff polymer chains, e.g. the wormlike chain model.

\section{Concluding remarks and perspectives}

Self-consistent field theory (SCFT) promises to be a concrete technique to capture all of the structural features of self-assembled complex foods at the equilibrium and constitutes a solid framework towards prediction of their topology at thermodynamic equilibrium. The establishment of an exact quantitative general predictive tool relies on a realistic parameterization of the physical interactions as well as the short-scale molecular details of the systems of interests. We are working aggressively towards improving the field-theoretic models that are used to describe selfassembly in lipid-water mixtures. Current efforts include the development of efficient numerical strategies for computing the single chain partition functions of short RIS chains and the incorporation of head group hydration models, hydrophobic effects, and other phenomena connected with the ability of the solvent to contribute hydrogen bonds. Finally, we are working on the incorporation of charged residues into polymeric fluids, which will enable a much wider variety of 'polyelectrolyte' systems to be described, such as phospholipids, peptides and charged polysaccharides. We are hopeful that in the near future, SCFT can become a truly predictive tool that is useful in unraveling the complex behavior of food grade polymers at equilibrium.

\section{References}

Barrat, J. L., Fredrickson, G. H., \& Sides, S. W. (2005). Introducing variable cell shape methods in field theory simulations of polymers. Journal of Physical Chemistry B, 109, 6694-6700.

Bekiranov, S., Bruinsma, R., \& Pincus, P. (1997). Solution behavior of polyethylene oxide in water as a function of temperature and pressure. Physical Review E, 55, 577-585. 
Ceniceros, H. D., \& Fredrickson, G. H. (2004). Numerical solution of polymer self-consistent field theory. Multiscale Modeling and Simulation, 2, 452-474.

Cochran, E. W., \& Bates, F. S. (2002). Thermodynamic behavior of poly(cyclohexylethylene) in polyolefin diblock copolymers. Macromolecules, 35, 7368-7374.

de Campo, L., Yaghmur, A., Sagalowicz, L., Leser, M. E., Watzke, H., \& Glatter, O. (2004). Reversible phase transitions in emulsified nanostructured lipid systems. Langmuir, 20, 5254-5261.

De Gennes, P. G. (1969). Some conformation problems for long macromolecules. Reports on Progress in Physics, 32, 187-206.

Dhinojwala, A., \& Granick, S. (1997). Relaxation time of confined aqueous films under shear. Journal of the American Chemical Society, 119, 241-242.

Doi, M., \& Edwards, S. F. (1988). The theory of polymer dynamics. Oxford: Oxford University Press.

Edwards, S. F. (1965). The statistical mechanics of polymers with excluded volumes. Proceedings of the Physical Society of London, 85, 613-624.

Flory, P. J. (1956). Statistical thermodynamics of semi-flexible chain molecules. Proceedings of the Royal Society of London Series A, Mathematical and Physical Sciences, 234, 60-73.

Fredrickson, G. H. (in press). The equilibrium theory of inhomogeneous polymers. Oxford University Press.

Fredrickson, G. H., Ganesan, V., \& Frolet, F. (2002). Field-theoretic computer simulation methods for polymers and complex fluids. Macromolecules, 35, 16-39.

Freed, K. (1972). Functional integrals and polymer statistics. Advances in Chemical Physics, 22, 1.

Granick, S. (1991). Motions and relaxations of confined liquids. Science, 253, 1374-1379.

Hamley, I. W. (1998). The physics of block copolymers. New York: Oxford University Press.

Hu, H. W., \& Granick, S. (1992). Viscoelastic dynamics of confined polymers melts. Science, 258, 1339-1342.

Israelachvili, J. N. (1991). Intermolecular and surfaces forces (2nd ed.). New York: Academic Press.

Jones, J. L., \& McLeish, T. C. B. (1995). Rheological response of surfactant cubic phases. Langmuir, 11, 785-792.

Jones, J. L., \& McLeish, T. C. B. (1999). Concentration fluctuations in surfactant cubic phases: Theory, rheology, and light scattering. Langmuir, 15, 7495-7503.

Jonsson, B., Lindman, B., Holmberg, K., \& Kronberg, B. (2001). Surfactants and polymers in aqueous solutions. Chichester, England: Wiley.

Khandpur, A. K., Forster, S., Bates, F. S., Hamley, I. W., Ryan, A. J., Bras, W., et al. (1995). Polyisoprene-polystyrene diblock copolymer phase diagram near the order-disorder transition. Macromolecules, 28, 8796-8806.

Krog, N. J. (1990). Food emulsifiers and their chemical and physical properties. In S. E. Friberg, \& K. Larsson (Eds.), Food emulsions (p. 141). New York: Marcel Dekker.

Li, X. J., \& Schick, M. (2001). Theory of tunable $\mathrm{pH}$-sensitive vesicles of anionic and cationic lipids or anionic and neutral lipids. Biophysical Journal, 80, 1703-1711.

Lindstrom, M., Ljusberg-Wahren, H., Larsson, K., \& Borgstrom, B. (1981). Aqueous lipid phases of relevance to intestinal fat digestion and absorption. Lipids, 16, 749-754.

Mariani, P., Rustichelli, F., Saturni, L., \& Cordone, L. (1999). Stabilization of the monoolein Pn $3 \mathrm{~m}$ cubic structure on trehalose glasses. European Biophysics Journal, 28, 294-301.
Matsen, M. W. (1995a). Stabilizing new morphologies by blending homopolymer with block copolymer. Physical Review Letters, 74, 4225-4228.

Matsen, M. W. (1995b). Phase behavior of block copolymer/homopolymer blends. Macromolecules, 28, 5765-5773.

Matsen, M. W., \& Bates, F. S. (1996). Unifying weak- and strongsegregation block copolymer theories. Macromolecules, 29, 1091-1098.

Matsen, M. W., \& Schick, M. (1994). Stable and unstable phases of a diblock copolymer melt. Physical Review Letters, 72, 2660-2663.

Matsyama, A., \& Tanaka, F. (1990). Theory of solvation-induced reentrant phase-separation in polymer-solutions. Physical Review Letters, 65, 341-344.

Mezzenga, R., Grigorov, M., Zhang, Z., Servais, C., Sagalowicz, L., Romoscanu, A. I., et al. (2005). Order-to-order transitions in lyotropic liquid crystals. Langmuir, 21, 6165-6169.

Mezzenga, R., Meyer, C., Servais, C., Romoscanu, A. I., Sagalowicz, L., \& Hayward, R. C. (2005). Shear rheology of lyotropic liquid crystals: A case study. Langmuir, 21, 3322-3333.

Mezzenga, R., Schurtenberger, P., Burbidge, A., \& Michel, M. (2005). Understanding foods as soft materials. Nature Materials, 4, 729-740.

Monduzzi, M., Ljusberg-Wahren, H., \& Larsson, K. R. (2000). A ${ }^{13} \mathrm{C}$ NMR study of aqueous dispersions of reversed lipid phases. Langmuir, 16, 7355-7358.

Muller, M., \& Schick, M. (1998). Calculation of the phase behavior of lipids. Physical Review E, 57, 6973-6978.

Pitzalis, P., Monduzzi, M., Krog, N., Larsson, H., Ljusberg-Wahren, H., \& Nylander, T. (2000). Characterization of the liquidcrystalline phases in the glycerol monooleate/diglycerol monooleate/water system. Langmuir, 16, 6358-6365.

Qiu, H., \& Caffrey, M. (2000). The phase diagram of the monoolein/water system: Metastability and equilibrium aspects. Biomaterials, 21, 223-234.

Rodriguez-Abreu, C., Garcia-Roman, M., \& Kunieda, H. (2004). Rheology and dynamics of micellar cubic phases and related emulsions. Langmuir, 20, 5235-5240.

Saturni, L., Rustichelli, F., Di Gregorio, G. M., Cordone, L., \& Mariani, P. (2001). Sugar-induced stabilization of the monoolein Pn3m bicontinuous cubic phase during dehydration. Physical Review E, 64 (art. no. 040902).

Schwarz, U. S., \& Gompper, G. (2001). Bending frustration of lipidwater mesophases based on cubic minimal surfaces. Langmuir, 17, 2084-2096.

Scriven, L. E. (1976). Equilibrium bicontinuous structure. Nature, 263, 123-125.

Sides, S. W., \& Fredrickson, G. H. (2003). Parallel algorithm for numerical self-consistent field theory simulations of block copolymer structure. Polymer, 44, 5859-5866.

Thies, M., Quitschau, P., Zimmermann, K., Rusch, V., Faunce, C. A., \& Paradies, H. H. (2002). Liquid-like ordered colloidal suspensions of lipid A: The influence of lipid A particle concentration. The Journal of Chemical Physics, 116, 3471-3483.

Vauthey, S., Milo, C., Frossard, P., Garti, N., Leser, M. E., \& Watzke, H. J. (2000). Structured fluids as microreactors for flavor formation by the Maillard reaction. Journal of Agricultural and Food Chemistry, 48, 4808-4816.

Yaghmur, A., de Campo, L., Sagalowicz, L., Leser, M. E., \& Glatter, O. (2005). Emulsified microemulsions and oil-containing liquid crystalline phases. Langmuir, 21, 569-577. 